\title{
Finite element exterior calculus with script geometry
}

Cite as: AIP Conference Proceedings 2116, 160003 (2019); https://doi.org/10.1063/1.5114147

Published Online: 24 July 2019

Paula Cerejeiras, Uwe Kähler, and Dmitrii Legatiuk

\section{ARTICLES YOU MAY BE INTERESTED IN}

Numerical modelling of an induction heating problem

AIP Conference Proceedings 2116, 160002 (2019); https://doi.org/10.1063/1.5114146

On quaternionic functions for the solution of an ill-posed Cauchy problem for a viscous fluid AIP Conference Proceedings 2116, 160005 (2019); https://doi.org/10.1063/1.5114149

Application of the hypercomplex fractional integro-differential operators to the fractional Stokes equation

AIP Conference Proceedings 2116, 160004 (2019); https://doi.org/10.1063/1.5114148

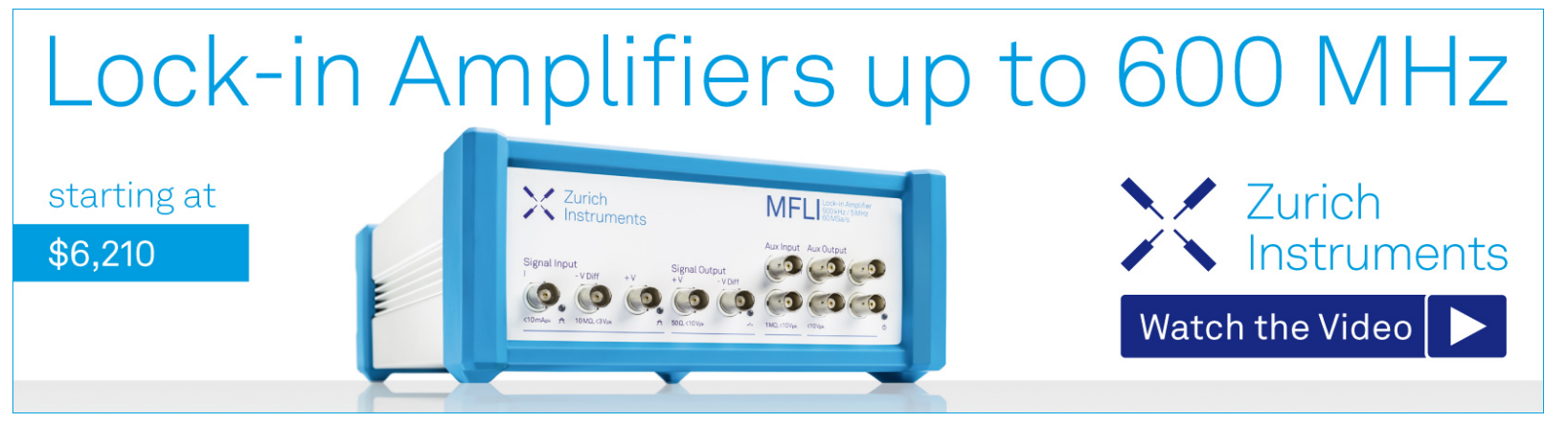




\title{
Finite Element Exterior Calculus with Script Geometry
}

\author{
Paula Cerejeiras ${ }^{1}$, Uwe Kähler ${ }^{1}$ and Dmitrii Legatiuk ${ }^{2, a)}$ \\ ${ }^{1}$ CIDMA - Center for $R \mathcal{E}$ D in Mathematics and Applications, Universidade de Aveiro, Portugal \\ ${ }^{2}$ Chair of Applied Mathematics, Bauhaus-Universität Weimar, Germany

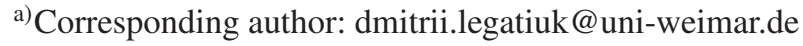

\begin{abstract}
Finite element method is probably the most popular numerical method used in different fields of applications nowadays. While approximation properties of the classical finite element method, as well as its various modifications, are well understood, stability of the method is still a crucial problem in practice. Therefore, alternative approaches based not on an approximation of continuous differential equations, but working directly with discrete structures associated with these equations, have gained an increasing interest in recent years. Finite element exterior calculus is one of such approaches. The finite element exterior calculus utilises tools of algebraic topology, such as de Rham cohomology and Hodge theory, to address the stability of the continuous problem. By its construction, the finite element exterior calculus is limited to triangulation based on simplicial complexes. However, practical applications often require triangulations containing elements of more general shapes. Therefore, it is necessary to extend the finite element exterior calculus to overcome the restriction to simplicial complexes. In this paper, the script geometry, a recently introduced new kind of discrete geometry and calculus, is used as a basis for the further extension of the finite element exterior calculus.
\end{abstract}

\section{INTRODUCTION}

The Finite Element Method (FEM) is probably the most popular numerical method used nowadays. The reason for such a popularity comes from several facts: (i) possibility to work with realistic geometries; (ii) flexibility to adopt the method to a specific problem by choosing an appropriate mesh and a desired regularity; (iii) a well-established mathematical basis for the classical version FEM, see [1]. The solution procedure with the FEM starts with the weak or variational formulation of a given boundary value problem, then a discretised problem is constructed by using a projection onto a finite-dimensional subspace. The finite-dimensional subspace is typically constructed based on the triangulation established over domain with the specific aim to obtain basis functions with smallest possible supports.

As most of approximation methods, the FEM approximates the continuous boundary value problem leading to the necessity of proving not only approximation quality by the FEM, but the stability of discretised problem as well. To overcome the stability issues, alternative approaches based on a direct work with discrete structures have become popular in recent years. By working on the discrete level, all important physical quantities, e.g. conservation laws, are satisfied directly on the discrete level. For example, methods based on finite differences, such as discrete potential theory [2], discrete function theory [3], theory of discrete analytic functions [4], and discrete Clifford analysis [5], have been the main object of interest for a long time. However, a discrete formulation of the FEM has been introduced quite recently with the development of the finite element exterior calculus.

Finite element exterior calculus has been introduced recently in works $[6,7]$. The main idea of the finite element exterior calculus is to utilise tools of algebraic topology, particularly de Rham cohomology and Hodge theory. Using the simplicial complexes as starting point, differential forms are utilised to formulate the continuous problem on the discrete level. As the result, the well-posedness of the discrete problem can be easily proved.

Despite of obvious advantages of the finite element exterior calculus, it still has some limitations coming from its restriction to simplicial complexes. Particularly, elements of more general shapes are often required in practical engineering applications, especially in the applications where a high accuracy of approximation of the geometry is needed, e.g. applications in aerospace engineering. To allow elements of more general shapes, the classical FEM has been extended in recent years, and so called, isogeometric analysis has been introduced [8]. Additionally, elements with curved boundaries appear in the case of a continuous coupling between the FEM and the analytical solution based

International Conference of Numerical Analysis and Applied Mathematics (ICNAAM 2018)

AIP Conf. Proc. 2116, 160003-1-160003-4; https://doi.org/10.1063/1.5114147

Published by AIP Publishing. 978-0-7354-1854-7/\$30.00 
on function-theoretic methods, see for example works [9, 10]. The need for curved-side elements arise naturally in this case, since function-theoretic methods allow to construct explicit solutions only for problems given in canonical domains, such as disks in $2 \mathrm{~d}$ and balls in $3 \mathrm{~d}$. Thus, further development of the finite element exterior calculus requires its extension to more general types of triangulations.

The objective of this paper is to present first steps in the extension of the finite element exterior calculus. Following the classical theory of FEM [1], at first it is necessary to introduce a more general geometrical setting than simplicial complexes. For this we are going to use ideas from a new kind of discrete geometry and calculus called script geometry, see [11].

\section{BASICS OF SCRIPT GEOMETRY}

Following [11], we recall in this section the basic notions of the script geometry, while all of necessary results related to the finite element exterior calculus will be introduced directly on the way of its extension to tight scripts in Section 3. We start with the notion of a script:

Definition 1 A script is a collection $\mathfrak{S}:=\left\{\mathfrak{S}_{-1}, \mathfrak{S}_{0}, \mathfrak{\Xi}_{1}, \ldots, \mathfrak{\Xi}_{k}, \ldots, \mathfrak{\Xi}_{m}\right\}$ of sets $\mathfrak{\Xi}_{k}$, the elements of which are called $k$-cells. In particular,

$$
\begin{aligned}
& \mathfrak{S}_{-1}:=\{\infty\}, \quad \Im_{0}:=\left\{p_{1}, \ldots, p_{j}, \ldots, p_{n_{0}}\right\}, \quad \Im_{1}:=\left\{l_{1}, \ldots, l_{j}, \ldots, l_{n_{1}}\right\}, \\
& \mathfrak{S}_{2}:=\left\{v_{1}, \ldots, v_{j}, \ldots, v_{n_{2}}\right\}, \quad \Im_{k}:=\left\{c_{1}^{k}, \ldots, c_{j}^{k}, \ldots, c_{n_{k}}^{k}\right\} .
\end{aligned}
$$

Traditionally 0, 1 and 2-cells are called points, lines and planes, respectively.

Definition $2 \quad$ A linear combination over $\mathbb{Z}$ of $k$-cells is called a $k$-chain:

$$
C_{k}:=\sum_{j} \lambda_{j} c_{j}^{k}, \quad \lambda_{j} \in \mathbb{Z},
$$

and we denote the module of $k$-chain by $\mathfrak{\leftarrow}_{k}$. The boundary map $\partial$ from $\mathfrak{\Xi}_{k}$ into $\mathfrak{C}_{k-1}$, the module of $(k-1)$-chains, is defined by:

$$
\partial c_{j}^{k}:=\sum_{s} \lambda_{j}^{k, s} c_{s}^{k-1},
$$

which naturally extends to the module $\mathfrak{C}_{k}$, and it is subject to $\partial^{2}=0$.

Definition $3 \quad A k$-chain $C_{k}$ for which $\partial C_{k}=0$, it is generally called a $k$-cycle. A $k$-chain

$$
C_{k}=\sum_{j} \lambda_{j}^{k} c_{j}^{k}
$$

for which $\lambda_{j}^{k}= \pm 1$ is called an oriented surface, or simply a surface. A surface $C_{k}$ for which $\partial C_{k}=0$ is a closed surface.

Definition 4 A script $\subseteq$ for which every cell boundary $\partial c_{j}^{k}$ is a closed surface is called a geoscript.

Definition $5 \quad$ A closed surface $C_{k}$ is called tight if and only iffor every closed surface $C_{k}^{\prime}$ with supp $C_{k}^{\prime} \subset \operatorname{supp} C_{k}$, it follows that $C_{k}^{\prime}= \pm C_{k}$, i.e. $C_{k}$ is the only closed surface, up to sign, with support inside $\operatorname{supp} C_{k}$.

Definition 6 A geomap $G: \Im \rightarrow \mathbb{S}^{\prime}$ between two tight geoscripts $\mathbb{S}_{\text {and }} \mathbb{\Xi}^{\prime}$ is a collection of linear maps $g_{k}: \Xi_{k} \rightarrow$ $\Im_{k}^{\prime}$ with the following two properties:

(i) the image of every $k$-surface $C_{k} \in \Xi_{k}$ is a $k$-surface $C_{k}^{\prime} \in \Im_{k}^{\prime}$, e.g. on a $k$-cell $c_{k}^{k}$ we have:

$$
g_{k}\left(c_{j}^{k}\right)=\sum \mu_{j}^{k, s} c_{s}^{\prime k}, \quad \mu_{j}^{k, s} \in\{-1,1\} .
$$

(ii) for each $k$, the natural extension of $g_{k}$ to a set of $k$-chains fulfills the relation $\partial g_{k}\left(C_{k}\right)=g_{k-1}\left(\partial C_{k}\right)$

Moreover, $g_{k}$ is called tight if it maps tight surfaces to tight surfaces. 


\section{FINITE ELEMENT METHOD WITH SCRIPTS}

Since our goal is to extend the finite element exterior calculus to script geometry, it is necessary to start from very basics of the finite element method. Thus, following [1] we start with a general description of a finite element space. Let us consider a domain $\Omega \subset \mathbb{R}^{n}$. Moreover, we assume that a mesh of tight scripts $\mathcal{F}_{h}$ is established over the set $\bar{\Omega}$, i.e. the set $\bar{\Omega}$ is subdivided into a finite number of tight scripts $\mathbf{K}$, called finite elements, in such a way that the following properties are satisfied:

(i) $\bar{\Omega}=\underset{\mathbf{K} \in \mathcal{F}_{h}}{\cup} \mathbf{K}$, where each $\mathbf{K} \in \mathcal{F}_{h}$ is a tight script.

(ii) Finite elements are not intersecting, and any face of a finite element $\mathbf{K}_{1}$ is either a face of another finite element $\mathbf{K}_{2}$, in which case the finite elements $\mathbf{K}_{1}$ and $\mathbf{K}_{2}$ are said to be adjacent, or a portion of the boundary $\Gamma$ of the set $\Omega$.

(iii) For each $\mathbf{K} \in \mathcal{F}_{h}$ the boundary $\partial \mathbf{K}$ is a closed surface.

Additionally, we denote now by $\Xi_{0}(\mathbf{K})$ and $\Xi_{d}(\mathbf{K})$ the 0 -cells and $n$-cells of a specific element $\mathbf{K}$, respectively, i.e. the set of vertices and the set of faces of dimension $d$. Moreover, we also define $\mathfrak{S}\left(\mathcal{F}_{h}\right):=\cup_{0 \leq d \leq n} \mathfrak{S}_{d}(\mathbf{K})$, the set of all faces in the mesh of tight scripts $\mathcal{F}_{h}$.

We would like to make an important remark: in contrast to the finite element exterior calculus [6,7], where only elements of a polyhedral shape are allowed, we consider here the finite elements of a more general shape: any shape allowed by a tight script, i.e. any shape allowed by definitions in Section 2. Moreover, by the construction, the elements are not required to be convex, and the only restriction is the absence of self-intersecting shapes, which is controlled by the restriction to tight scripts.

Establishing of a finite element scheme based on tight scripts requires the notion of the coderivative $\delta: \mathbf{X}^{k}(\Omega) \rightarrow$ $\mathbf{X}^{k-1}(\Omega)$, which is defined as follows

$$
\star \delta \omega:=(-1)^{k} d \star \omega, \quad \omega \in \mathbf{X}^{k}(\Omega),
$$

where $\star$ is the Hodge star operator [6]. By application of the Stokes theorem, it is possible to show that the coderivative operator $\delta$ is a formally adjoint of the exterior derivative operator $d$, i.e. $d=\delta^{*}$. Now, we can introduce the following Hilbert spaces:

and its dual

$$
H^{*} \mathbf{X}^{k}:=\left\{u \in L^{2} \mathbf{X}^{k} \mid \delta u \in L^{2} \mathbf{X}^{k+1}\right\},
$$

$$
H \mathbf{X}^{k}:=\left\{u \in L^{2} \mathbf{X}^{k} \mid d u \in L^{2} \mathbf{X}^{k+1}\right\},
$$

where $L^{2} \mathbf{X}^{k}$ and $L^{2} \mathbf{X}^{k+1}$ are spaces of differential $k$ - and $(k+1)$-forms on tight scripts with $L^{2}$ coefficients. These spaces are related by a well-known relation $H^{*} \mathbf{X}^{k}=\star H \mathbf{X}^{k-n}$. Considering that $d=d^{k}$ is the exterior derivative operator taking differential $k$-forms to differential $(k+1)$-forms, and all the $d^{k}$ form the $L^{2}$ de Rham complex

$$
0 \longrightarrow H \mathbf{X}^{0} \stackrel{d^{0}}{\longrightarrow} H \mathbf{X}^{1} \stackrel{d^{1}}{\longrightarrow} \cdots \stackrel{d^{n-1}}{\longrightarrow} H \mathbf{X}^{n}
$$

as in the finite element exterior calculus case. The dual complex can be written similarly, and essentially contains the same information. Now, we define a finite element space $\mathbf{X}_{h}$, which is a finite-dimensional space of differential forms with $L^{2}$ coefficients. To characterise the finite element space we have to specify local spaces (spaces over specific finite elements) and degrees of freedom:

1. Let $v_{h}$ be a set of all functions belonging to the space $\mathbf{X}_{h}$, then we define the local spaces $\mathbf{P}_{\mathbf{K}}$ of the functions (shape functions) over the finite elements $\mathbf{K} \in \mathcal{F}_{h}$ by the restrictions $\mathbf{P}_{\mathbf{K}}=\left\{v_{h \mid \mathbf{K}}: v_{h} \in \mathbf{X}_{h}\right\}$.

2. Let $\Sigma$ denote the finite set of linearly independent continuous linear functionals $\mathbf{X}_{h} \rightarrow \mathbb{R}$, called degrees of freedom, which unisolvent in the sense of P.G. Ciarlet [1], and such that each degree of freedom is associated to a specific $n$-cell of $s \in \mathfrak{S}\left(\mathcal{F}_{h}\right)$. Moreover, the degrees of freed of two adjacent finite elements $K_{1}$ and $K_{2}$ are in a specific 1-to-1 correspondence.

The notion of affine-equivalent sets plays a crucial role in the finite element method, particularly for the error analysis. Therefore, it is necessary to introduce a similar notion for the finite element method with script geometry. Particularly, we extend the definition of a geomap presented in [11] as follows:

Definition $7 \quad T$ wo finite elements $\hat{K}$ and $K$, with the corresponding local finite element spaces $\hat{P}, P$, and the associated degrees of freedom $\hat{\Sigma}, \Sigma$, are said to be geomap-equivalent if the following conditions hold: 
(i) the geomap $G: \Im(\hat{K}) \rightarrow \Im(K)$ is an isomorphism between the tight scripts of these elements, i.e. $\Xi(\hat{K})$ and $\Im(K)$;

(ii) $K=G(\hat{K})$;

(iii) $P=\left\{p: K \rightarrow \mathbb{R} ; \quad p=\hat{p} \cdot G^{-1}, \quad \hat{p} \in \hat{P}\right\}$;

(iv) $\Sigma=G(\hat{\Sigma})$,

where the action of the geomap $G$ on degrees of freedom implies the action on the corresponding cells of the finite elements.

\section{SUMMARY AND OUTLOOK}

In this short paper we have presented first steps in the extension of the finite element exterior calculus to the script geometry, which is a recently introduced new kind of discrete geometry and calculus. Particularly, meshes of tight scripts allowing very general shapes of finite elements have been introduced. Moreover, the notion of geomap-equivalence for finite elements based on tight scripts has been introduced. This equivalence notion is the basis of future work related to error analysis of the proposed scheme. Additionally, a stable discrete variational formulation in the framework of the finite element method with script geometry will be discussed in future.

\section{ACKNOWLEDGMENTS}

The work of the third author is supported by the project Neue funktionentheoretische Methoden für instationäre PDE, funded by Programme for Cooperation in Science between Portugal and Germany, DAAD-PPP Deutschland-Portugal, Ref: 57340281. The work of the first and second authors is supported via the project "New Function Theoretical Methods in Computational Electrodynamics" approved under the agreement Ações Integradas Luso-Alemãs DAAD-CRUP, ref. A-15/17. Additionally, we would like to thank the Erasmus+ programme "ERASMUS+ Strategic Partnership Forecast Engineering: From Past Design to Future Decisions, 2016-1-DE01-KA203-002905". The work of the first and the second author was supported by Portuguese funds through the CIDMA - Center for Research and Development in Mathematics and Applications, and the Portuguese Foundation for Science and Technology ("FCT-Fundação para a Ciência e a Tecnologia"), within project UID/MAT/0416/2013. The work of the third author has been supported via the Seed Fund for young researchers from the Bauhaus-Universität Weimar.

\section{REFERENCES}

[1] P.G. Ciarlet, The finite element method for elliptic problems, North-Holland Publishing Company, 1978.

[2] V.S. Ryaben'kii, Method of difference potentials and its applications, Springer-Verlag, 2002.

[3] K. Gürlebeck, A. Hommel, On finite difference potentials and their applications in a discrete function theory, Mathematical Methods in the Applied Sciences, 25, 2002, 1563-1576.

[4] L. Lovász, Discrete analytic functions: an exposition, Surveys in Differential Geometry, Volume 9, pp. 241273, 2004.

[5] F. Brackx, H. De Schepper, F. Sommen, L. Van de Voorde, Discrete Clifford analysis: a germ of function theory, Hypercomplex Analysis, Birkhäuser Basel, 2009, 37-53.

[6] D.N. Arnold, R.S. Falk, R. Winther, Finite element exterior calculus, homological techniques, and applications, Acta Numerica, pp. 1-155, 2006.

[7] D.N. Arnold, R.S. Falk, R. Winther, Finite element exterior calculus: from Hodge theory to numerical stability, Bulletin (New Series) of the American Mathematical Society, 47(2), pp. 281-354, 2010.

[8] Y. Bazilevs, L. Beirão da Veiga, J.A. Cottrell, T.J.R. Hughes, G. Sangalli, Isogeometric analysis: approximation, stability and error estimates for h-refined meshes, Mathematical Models and Methods in Applied Sciences, 16(7), 2006.

[9] K. Gürlebeck, U. Kähler, D. Legatiuk, Error estimates for the coupling of analytical and numerical solutions. Complex Analysis and Operator Theory, 11(5), pp. 1221-1240, 2017.

[10] K. Gürlebeck, D. Legatiuk, On the continuous coupling of finite elements with holomorphic basis functions. Hypercomplex Analysis: New perspectives and applications, ISBN 978-3-319-08770-2, Birkhäuser, Basel, 2014.

[11] P. Cerejeiras, U. Kähler, F. Sommen, A. Vajiac, Script geometry, Modern Trends in Hypercomplex Analysis, pp. 79-110, 2016. 